\section{Juristinnen fragen - Politiker(innen) antworten}

\section{Entgelt(un)gleichheit: Der deutsche „Gender Pay Gap“ von fast 25 Prozent}

\section{Donnerstag, 25. November 2010, 17.00 Uhr in der Vertretung des Freistaats Thüringen beim Bund Mohrenstraße 64, 10117 Berlin}

Anmeldung: Bitte bis 17. November 2010 schriftlich und verbindlich (E-Mail: geschaeftsstelle@djb.de, Anschrift: Deutscher Juristinnenbund, Anklamer Straße 38, 10115 Berlin, Fax: 030 44327022) bei der djb-Geschäftsstelle.

Überall in Europa verdienen Frauen weniger als Männer. In den Mitgliedstaaten der Europäischen Union betrug der geschlechtsbezogene Einkommensunterschied („Gender Pay Gap“) im Jahr 2007 im Durchschnitt 17,6 Prozent. In Deutschland liegt er derzeit sogar über 23 Prozent und damit fast bei einem Viertel. Unter den 27 EU-Mitgliedstaaten lag Deutschland im Jahr 2007 weit hinten auf Platz 21.

Für den Gender Pay Gap gibt es verschiedene Gründe. Einer davon ist Entgeltdiskriminierung, also der Verstoß gegen den Rechtsanspruch auf gleiches Entgelt für Frauen und Männer bei gleicher oder gleichwertiger Arbeit. Dies steht im Widerspruch sowohl zum Grundgesetz als auch zum Unionsrecht sowie dem deutschen AGG. Dennoch hat diese klare rechtliche Lage bisher wenig geändert. Es bedarf offensichtlich unterstützender rechtlicher Maßnahmen. Vorschläge hierzu liegen vor; sie reichen von der Schaffung von Informations- und Auskunftsrechten der Beschäftigten und ihrer Vertretungen bis hin zu einem Verfahrensgesetz, das die tariflichen und betrieblichen Akteurinnen und Akteure wirksam in die Pflicht nimmt.

\section{Programm}

Begrüßung Jutta Wagner, Präsidentin des djb, Berlin

Statement Johanna Arenhövel, Beauftragte für die Gleichstellung von Frau und Mann beim Thüringer Ministerium für Soziales, Familie und Gesundheit, Erfurt

Vortrag Rechtsanwältin Prof. Dr. Marlene Schmidt, Frankfurt/Main

\section{Auf dem Podium}

Andrea Voßhoff MdB Rechtspolitische Sprecherin der CDU/ CSU-Bundestagsfraktion

Christel Humme MdB Sprecherin der Arbeitsgruppe Gleichstellungspolitik der SPD-Bundestagsfraktion

Sibylle Laurischk MdB Mitglied der FDP-Bundestagsfraktion, Vorsitzende des Ausschusses für Familie, Senioren, Frauen und Jugend des Deutschen Bundestages

Cornelia Möhring MdB Frauenpolitische Sprecherin der Bundestagsfraktion Die Linke, Mitglied im Ausschuss für Familie, Senioren, Frauen und Jugend des Deutschen Bundestages

Ekin Deligöz MdB Stellv. Vorsitzende der Bundestagsfraktion Bündnis 9o/Die Grünen, Mitglied im Ausschuss für Familie, Senioren, Frauen und Jugend des Deutschen Bundestages

Prof. Dr. Heide Pfarr (Moderation) Wissenschaftliche Direktorin des Wirtschafts- und Sozialwissenschaftlichen Instituts in der Hans-Böckler-Stiftung, Düsseldorf

ca. 19.30 Uhr, Imbiss im Dachgeschoss der Landesvertretung

\title{
Impressum
}

\section{Schriftleitung:}

Anke Gimbal, Rechtsassessorin (V.i.S.d.P.)

Juliane Lindner

\section{Redaktionsanschrift:}

Deutscher Juristinnenbund e. V.

Anklamer Str. 38

10115 Berlin

Telefon: 030 443270-0

Telefax: $030443270-22$

E-Mail: geschaeftsstelle@djb.de

\section{Druck und Verlag:}

Nomos Verlagsgesellschaft mbH \& Co. KG

Waldseestr. 3-5

D-76530 Baden-Baden

Telefon: 07221 2104-O

Telefax: 07221 2104-27
Anzeigenverwaltung und

Anzeigenannahme:

Sales friendly, Verlagsdienstleistungen

Bettina Roos

Siegburgerstr. 123

D-53229 Bonn

Telefon: 0228 97898-o

Telefax: 0228 97898-20

E-Mail: roos@sales-friendly.de

Die Zeitschrift sowie alle in ihr enthaltenen einzelnen Beiträge und Abbildungen sind urheberrechtlich geschützt. Jede Verwertung, die nicht ausdrücklich vom Urheberrechtsgesetz zugelassen ist, bedarf der vorherigen Zustimmung des Verlages.

Namentlich gekennzeichnete Artikel müssen nicht die Meinung des Herausgebers oder der Schriftleitung wiedergeben. Unverlangt eingesendete Manuskripte - für die keine Haftung übernommen wird - gelten als Veröffentli- chungsvorschlag zu den Bedingungen des Verlags. Es werden nur unveröffentlichte Originalarbeiten angenommen. Die Verfasser erklären sich mit einer nicht sinnentstellenden redaktionellen Bearbeitung einverstanden.

Erscheinungsweise:

vierteljährlich

Bezugspreis 2010:

jährlich 48,-€, Einzelheft 13,-€

Alle Preise zzgl. Vertriebs-Direktbeordnungsgebühren inkl. MWSt.;

Bestellungen nehmen entgegen:

Der Buchhandel und der Verlag; Kündigung jeweils drei Monate zum Kalenderjahresende. Zahlungen jeweils im Voraus an:

Nomos Verlagsgesellschaft, Postbank Karlsruhe, Konto 7363651 (BLZ 66010075) oder Stadtsparkasse Baden-Baden, Konto 5002266 (BLZ 66250030). 